\title{
Traumatic arteriovenous fistula of the left parotid region: A case report
}

\author{
CHENXING WANG, YANENG GE and LONGJIANG LI \\ Department of Oncology, West China Hospital of Stomatology, Sichuan University, Chengdu, Sichuan 610041, P.R. China
}

Received January 27, 2015; Accepted December 2, 2015

DOI: $10.3892 / 01.2016 .4371$

\begin{abstract}
The formation of arteriovenous fistula beneath the parotid is relatively rare. To the best of our knowledge, the present study reports the first case of a 48-year-old man with a pulsatile mass on the left parotid region, which had been present for $>3$ years following penetrating wounds. Computed tomography angiography and digital subtraction angiography revealed a fistula between the left external carotid artery and the left jugular vein. The patient was successfully managed through surgical excision without embolization. Intraoperative facial nerve monitoring was employed to prevent postoperative facial paralysis. The present study briefly reviewed the current literature, illustrating the pathophysiology, symptoms, imaging studies and treatment for arteriovenous fistula in the head and neck region. To conclude, arteriovenous fistula located near the facial nerve may be successfully managed by surgical treatment, without permanent facial paralysis.
\end{abstract}

\section{Introduction}

Arteriovenous fistulas (AVFs), are lesions with a single abnormal connection between an artery and a vein that bypass the capillary system. AVFs are distinct from arteriovenous malformations (AVMs), which are disfiguring lesions with numerous arteriovenous connections (1). Arteriovenous fistulas may occur spontaneously or may be caused by trauma or surgery (2). AVFs may be acquired following penetrating or blunt trauma, such as car accident injuries or stab wounds (3-5). Although the head and neck tissues are relatively vascularized, AVFs located in this region are relatively rare, and to the best of our knowledge, no AVF of the parotid region has previously been reported in the literature $(3,6,7)$. Incidence and mortality rates vary depending on the blood vessels affected by AVFs,

Correspondence to: Professor Longjiang Li, Department of Oncology, West China Hospital of Stomatology, Sichuan University, 14 Renmin South Road, Chengdu, Sichuan 610041, P.R. China E-mail: lilongjiang_scu@163.com

Key words: arteriovenous fistula, parotid region, facial nerve, traumatic as well as the time elapsed prior to treatment (4). Cases of AVF involving the neck area treated with both endovascular embolization and surgical resection have been reported (4). AVFs have been successfully managed surgically or with embolization, however, it is challenging to predict the optimal treatment method $(2,3)$

The present study reports the case of a patient with an arteriovenous fistula of the left parotid region that was located between the left external jugular vein and the left external carotid artery, which occurred subsequent to a penetrating injury.

\section{Case report}

A 48-year-old man was referred to the Department of Oncology of West China Hospital of Stomatology (Chengdu, Sichuan, China) due to a pulsatile mass located on the left side of the face, which had been present for 3 years.

The patient's medical history included a penetrating trauma to his left facial region that occurred 8 years prior to the appearance of the slowly growing mass. The patient had undergone an embolization procedure at West China Hospital (Chengdu, China) 5 months previously in order to block the fistula between the branches of the external jugular vein and the external carotid artery. However, no evident recession was observed following the procedure and the patient was referred to the Department of Oncology, West China Hospital of Stomatology for further treatment.

A physical examination identified a soft, compressible and pulsatile mass located at the left parotid region. Auscultation over the lesion identified a murmur-like bruit. Computed tomography angiography (CTA) of the head and neck region was performed to identify the nature of the lesion and the feeding vessels. The CTA revealed an AVF on the left posterior ramus region that measured $4.0 \times 4.5 \mathrm{~cm}$ in diameter and was fed by a branch of the external carotid artery (Fig. 1). The identification was corroborated by digital subtraction angiography (DSA) (Fig. 2) and three-dimensional-DSA. The pre-surgery examination did not reveal any evident heart problems.

Since the patient had a history of failed embolization treatment, a surgical excision was performed without preoperative embolization. The surgical excision of the AVF was performed under general anesthetic, using an S-shape incision anterior to the tragus. Due to the close association between 


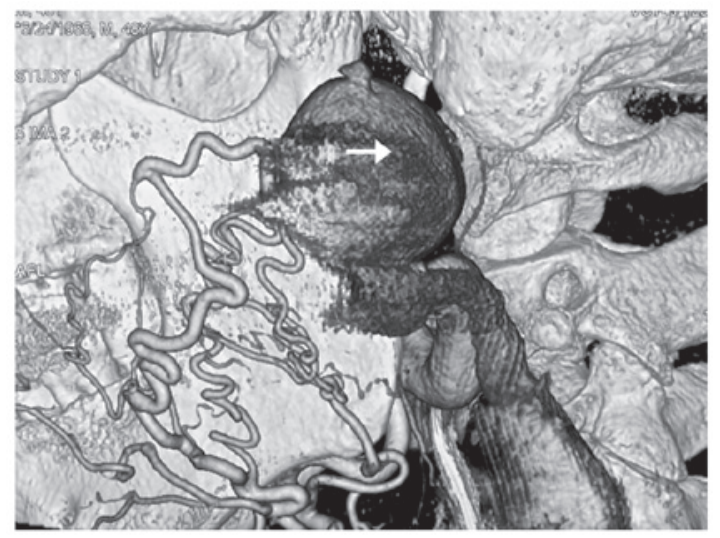

Figure 1. Lateral view of the computed tomography angiography identified the arteriovenous fistula (white arrow) located in the left parotid region and near the posterior border of the ramus.

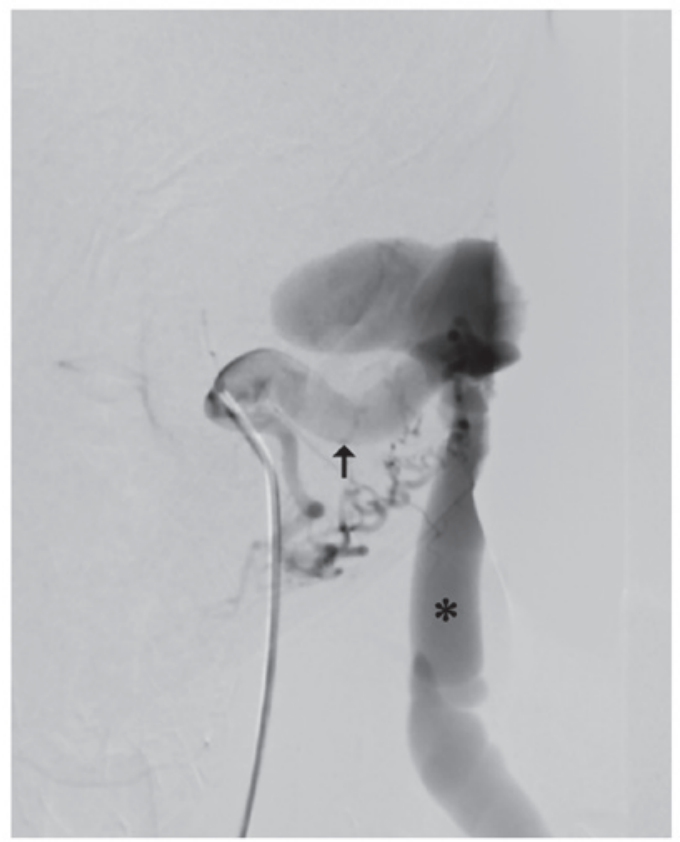

Figure 2. Left external carotid artery angiogram demonstrated early venous filling, identified the fistula (arrow) and the dilated left external jugular vein (asterisk).

the facial nerve and the mass, intraoperative facial nerve monitoring was employed during surgery for identifying the facial nerve. A partial parotidectomy was performed to expose the mass, while the facial nerve was protected and gently retracted aside. The feeding vessels were ligated prior to extirpation of the mass. Following ligation, the pulsation disappeared and the mass was wholly excised. The left parotid tissue was then reapproximated and the overlying skin was closed primarily. Postoperatively, the cosmetic appearance was satisfying and the patient was free from recurrence for one year subsequent to surgery.

The present patient provided written informed consent, and the study was performed in compliance with the Declaration of Helsinki and was approved by the Ethics Committee of the West China College of Stomatology (Sichuan University, Chengdu, Sichuan, China).

\section{Discussion}

AVFs are vascular lesions with a single connection between an artery and a vein, and are commonly attributed to previous trauma that affected the region, including penetrating wounds (4), blunt trauma (1) or surgery (8). AVF is defined as a lesion that has a single communication between an artery and a vein. Therefore, AVF is a distinct clinical entity from a congenital or acquired arteriovenous malformation (AVM), which has multiple distinct connections.

AVF symptoms may vary depending on the location and size of the fistula. Local symptoms are caused by disturbances to vessel system resistance, and may include the absence of reversal of diastolic flow, reversal of flow in the distal vessel, dilatation of the proximal venous segment, venous hypertension and incompetent valves. As a fistula enlarges, peripheral blood pressure changes often occur, and may be followed by heart failure (9).

AVF may be identified by a palpable thrill, auscultated bruit over the lesion and by the Branham-Nicoladoni sign (10); however, imaging continues to be required for the confirmation and precise location of the fistula.

While duplex ultrasonography, magnetic resonance angiography and CTA are practical for the diagnosis of AVF and may provide important information on the surrounding anatomical structures, DSA is the standard technique for imaging AVFs (9). DSA is particularly useful for surgical planning and for identifying the feeding and draining vessels. In the present study, CTA was used to confirm the presence and nature of the lesion as an AVF, to identify the morphology and surrounding tissue and to assist with therapeutic planning.

The goal of AVF treatment is to remove abnormal connections and to restore the normal anatomy and function of the artery and vein. AVFs of the external carotid system have previously been managed surgically (3) and with embolization (11). Clinically, it is challenging to predict the optimal treatment to ensure long-term success. Surgical treatment remains preferable in young patients with AVF of the extremities, unless the lesions are challenging to access (9). Endovascular techniques are particularly useful for patients who have comorbidities or lesions that are challenging to access.

In the present study, surgical therapy was determined to be the optimal treatment approach for several reasons. The embolization previously performed on the curent patient did not result in an evident recession of the mass. Without surgical intervention, a firm mass would remain in the parotid salivary region. While this may be an acceptable result in other, less conspicuous, areas of the body, the highly visible nature of the salivary region made the mass easily visible. As the mass was located beneath the parotid and near the facial nerve, it was challenging to expose and resect the mass while preventing facial paralysis. In the present study, intraoperative facial nerve monitoring was employed to fulfill the purpose of preventing facial paralysis.

To conclude, the present study reports the successful surgical treatment of a patient with a rare fistula of the parotid region, located between the external carotid artery and external jugular vein. The present study demonstrates that, with the aid of intraoperative facial nerve monitoring, surgical treatment is a viable option for the successful management of AVF lesions 
near the facial nerve following prior embolization procedures that fail to block the fistula.

\section{Acknowledgements}

The authors thank Dr Zhuoyuan Zhang (Department of Oncology, West China College of Stomatology, Sichuan University, Chengdu, China), who provided the qualified pictures of the patient and provided important suggestions for the manuscript.

\section{References}

1. Zer Toros S, Zorlu A, Deveci I, Deveci HS, Naiboglu B and Gökçeer T: Traumatic arteriovenous fistula of the upper lip: A case report. J Craniomaxillofac Surg 38: 485-487, 2010.

2. Nikfarjam J, Taub PJ, Patel A and Rose E: Arteriovenous fistula following radial forearm free flap. J Reconstr Microsurg 27 295-298, 2011.

3. Dell'Amore A, Castriota F, Calvi S, Magnano D, Noera G and Lamarra M: Post-traumatic carotid-jugular arterio-venous fistula. Heart Lung Circ 18: 293, 2009.
4. Robbs JV, Carrim AA, Kadwa AM and Mars M: Traumatic arteriovenous fistula: Experience with 202 patients. Br J Surg 81: 1296-1299, 1994.

5. Sanborn MR, Nasrallah I, Stanton DC, Stiefel MF, Hurst RW and Pukenas BA: Acquired arteriovenous fistula associated with traumatic oroantral fistula: Endovascular treatment. Head Neck 35: E138-E141, 2013.

6. Kalt M, Knipping L and Mangold G: Traumatic arteriovenous fistula between superior thyroid artery and vein. Chirurg 68: 1,304-1,306, 1997 (In German).

7. Adame N Jr and Bayless P: Carotid arteriovenous fistula in the neck as a result of a facial laceration. J Emerg Med 16: 575-578, 1998.

8. Chaloupka JC, Kibble MB and Hoffman JC: Ascending pharyngeal artery-internal jugular vein fistula complicating radical neck dissection. Neuroradiology 34: 524-525, 1992.

9. Sexton JA and Ricotta JJ: Endovascular approaches to arteriovenous fistula. Adv Surg 45: 83-100, 2011

10. Martin TJ, Hacein-Bey L and Rhee JS: Arteriovenous fistula of the lower lip: Case report of combined intravascular and surgical cure. WMJ 105: 47-50, 2006.

11. Regina G, Impedovo G, Angiletta D, Marotta V, Lillo A, Pestrichella F and De Blasi R: A new strategy for treatment of a congenital arteriovenous fistula of the neck. Case report. Eur J Vasc Endovasc Surg 32: 107-109, 2006. 